\title{
Different Approaches of Employing Cholesteric Liquid Crystals in Dye Lasers
}

\author{
Guram Chilaya ${ }^{1}$, Andro Chanishvili ${ }^{1}$, Gia Petriashvili ${ }^{1}$, Riccardo Barberi ${ }^{2}$, Maria Penelope de Santo ${ }^{2}$, \\ Mario Ariosto Matranga ${ }^{2}$
}

${ }^{1}$ Institute of Cybernetics, Euli str. 5, Tbilisi, Georgia; ${ }^{2}$ CEMIF.CAL, INFM CNR-Licryl Lab., Physics Department, University of Calabria, Rende (Cs), Italy.

Email: chilaya@yahoo.com

Received June $1^{\text {st }}, 2010$; revised December $9^{\text {th }}, 2010$; accepted January $28^{\text {th }}, 2011$.

\begin{abstract}
Two ways of employing cholesteric liquid crystals in tunable dye lasers are considered: the cholesterics as distributed feedback medium and the cholesterics as resonator mirrors. In the dye doped distributed feedback cholesteric liquid crystal lasers the frequency tuning is achieved exploiting light induced effects or using a specially designed cell assembling a chiral dopant concentration gradient in combination with suitable distribution of different dyes. Another approach represents the lasing in a multilayer system consisting of a dye doped isotropic solvent sandwiched between two CLC layers.
\end{abstract}

Keywords: Liquid Crystals, Lasers

\section{Introduction}

Cholesteric Liquid Crystals (CLCs) possess several unique properties: a supramolecular periodic helical structure due to the chirality of the molecules (the period - spiral pitch $\mathrm{P}$ - can be set in a wide range from $100 \mathrm{~nm}$ up to infinity), $100 \%$ selective reflection of circularly polarized light and the ability to control selective reflection wavelength, changing external or internal factors (electric, magnetic and acoustic fields, temperature, local order $[1,2]$. The maximum wavelength $\lambda_{0}$ and the spectral width $\Delta \lambda$ of the selective reflection band are equal to $\lambda_{0}$ $=\mathrm{P} \mathrm{n}$ and $\Delta \lambda=\mathrm{P} \Delta \mathrm{n}$ accordingly, where $\mathrm{n}$ is the average index of refraction and $\Delta \mathrm{n}$ is the birefringence of a layer perpendicular to the helix axis. According to the modern conceptions the CLCs can be considered as a one dimensional (1D) photonic crystal $[3,4]$. The existence of the selective reflection band and the ability to change smoothly the selective reflection wavelength over a wide range under the action of applied external forces make it possible to use in tunable dye lasers. The organic dye lasers have the distinction of being the first broadly tunable lasers and one of the most versatile coherent light sources. These lasers provide broad tunability over a spectral range that covers the ultraviolet (UV) to near infrared (NIR). Organic dye lasers were discovered in $1966[5,6]$.
Any conventional laser needs two necessary conditions to operate: the presence of a positive feedback and the presence of an active medium. As feedback elements usually resonator mirrors are used. The use of a CLC layer as a mirror in the dye lasers was proposed long time ago. The temperature dependence of the selective reflection band was exploited to tune a broad band dye laser [7].

But there is a class of lasers in which the external feedback elements are absent and the active medium acts as a distributed feedback, in this case the active medium must have a spatial periodic structure. It is important for development of compact lasers that the major functional elements of a mirror-less laser are combined in one cell: active medium, cavity, and tunable selector. There are different methods to create periodic structures, but the optimum is reached when the medium itself possesses a ready periodic structure with required parameters. In this respect CLCs are among the materials able to be used as active medium in Distributed Feedback (DFB) lasers - if the CLC consists of luminescent molecules or it contains a luminescent dopant, it becomes possible to build a tunable dye doped (DD) DFB laser. This approach allows solving one of the most actual problems in laser technique i.e. the creation of a simply and smoothly tunable laser. 
In this review paper two methods to obtain DD CLC DFB laser tuning will be presented. In the first approach, the frequency tuning is achieved by exploiting light induced effects. In the second method the spatial tuning of the laser emitted wavelength was distinguished with the use of a specially designed cell combining a chiral dopant concentration gradient and a suitable distribution of different dyes. Also lasing in a multilayer system consisting of a dye doped isotropic solvent sandwiched between two CLC cells will be considered.

\section{Light Tunable DDDFB CLC Lasers}

One of the most exciting developments in LC science and technology is the possibility of using light to control the parameters of a material $[8,9]$. We considered low molar mass calamatic thermotropic CLCs. According to the chemical nature of the constituent molecules CLC subdivided in the following ways: 1) Steroids, mainly cholesterol esters and their mixtures with each other; from which derives the name CLC; 2) Non-steroidal cholesterics (so-called chiral nematics); 3) Induced cholesteric systems comprising a nematic matrix and a liquidcryastalline or non-liquidcryastalline optically active dopant (OAD). It is known that the pitch in induced cholesteric systems depends on the structure of both the nematic host and the chiral dopant [10-13].

Reversible and irreversible changes in the helical pitch have been observed in CLC mixtures as a result of photochemical transformation of conformationally active components. In these mixtures, either a chiral component or a nematic component, or both components, can be photosensitive. Special photosensitive (chiral or achiral) compounds can be added to photoinsensitive chiral nematic mixtures. It should be noted that non-photosensitive mixture components should be transparent in the visible and near-UV regions. Investigations of the absorption edge spectra of nematic liquid crystals with different rigid fragments and bridging groups [14] have revealed that the nematic liquid crystals based on cyclohexylcyclohexanes are transparent in the visible and UV spectral regions up to $200 \mathrm{~nm}$ (Figure 1).

In our experiments we used mostly the nematic mixture ZLI-1695 (Merck) as a nematic host, which is the mixture of cyclohexylcyclohexanes

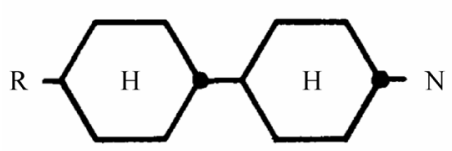

$\mathrm{R}=\mathrm{C}_{2}, \mathrm{C}_{3}, \mathrm{C}_{4}, \mathrm{C}_{7}$ with nematic range $13-72^{\circ} \mathrm{C}$.

A large number of papers investigating pitch control in CLC mixtures have been devoted to systems consisting

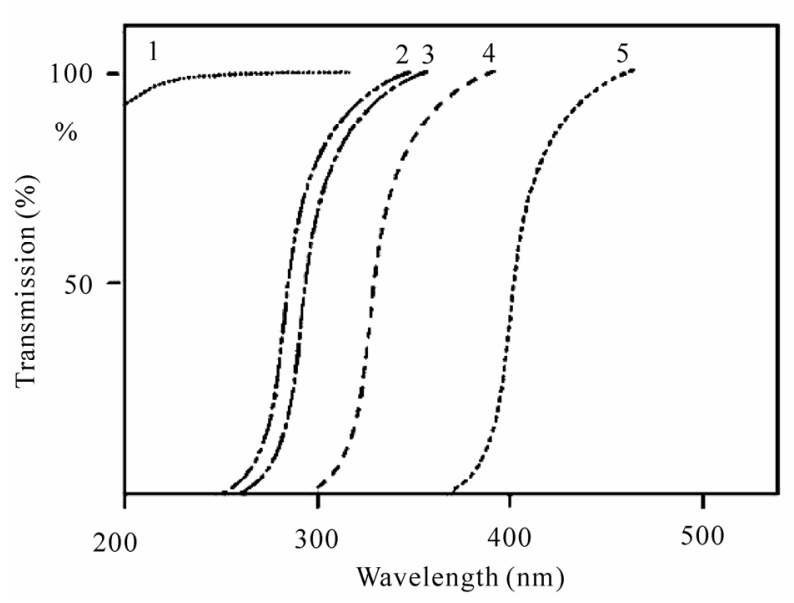

Figure 1. Absorption edge of liquid crystals: 1: Cyclohexylcyclohexanes, 2: Phenylcyclohexanes, 3: Esters, 4: Biphenyls, 5: Shiff bases. Layer thickness $=10 \mu \mathrm{m}$.

of conformationally active molecules, which are capable of light-induced trans-cis isomerization. Typically an elongated rod-like molecule (trans isomer) transforms under the influence of UV radiation to a bent or fractured form (cis isomer).

Azoxy-based (ZhK 440, NIOPIK: (2/3 p-n butyl-pmethoxyazoxybenzene $+1 / 3$ p-n-butyl-p-heptanoylazoxybenzene) LCs are transparent in a wider part of a spectrum than azo-compounds (ZhK 286, NIOPIK: $p$-ethoxyphenyl-p0-azophenyl hexanoate) as shown in Figure 3 when a small concentration of the compounds was dissolved in a transparent nematic.

In recent years, nematic liquid crystals themselves have been used as a photoizomerizable component in the study of the light-induced phenomena in chiral nematic mixtures. Usually the amount of photoactive material in these systems is small. More recently, CLC mixtures with a photoisomerizable nematic component typically comprising $60-80 \mathrm{wt} \%$ of the sample, with the rest of the sample being a photoinactive chiral compound has been investigated [15-21]. Different nematic liquid crys- tals based on azo and azoxy compounds, as well as on cinnamic acids, which can undergo photoisomerization, were investigated.

The color change of CLC mixtures under UV irradiation can be used for realization of a solar UV-B irradiation sensor. For example, the mixture based on a photosensitive derivative of cinnamate [22-24] has the absorp-

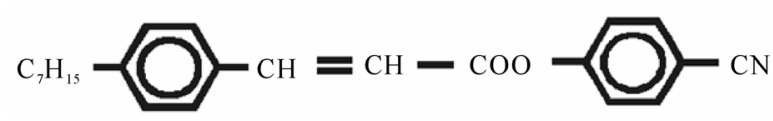

tion has the spectrum very close to the spectrum of human skin sensitivity to sunburn (UV-B range: $290-300$ 


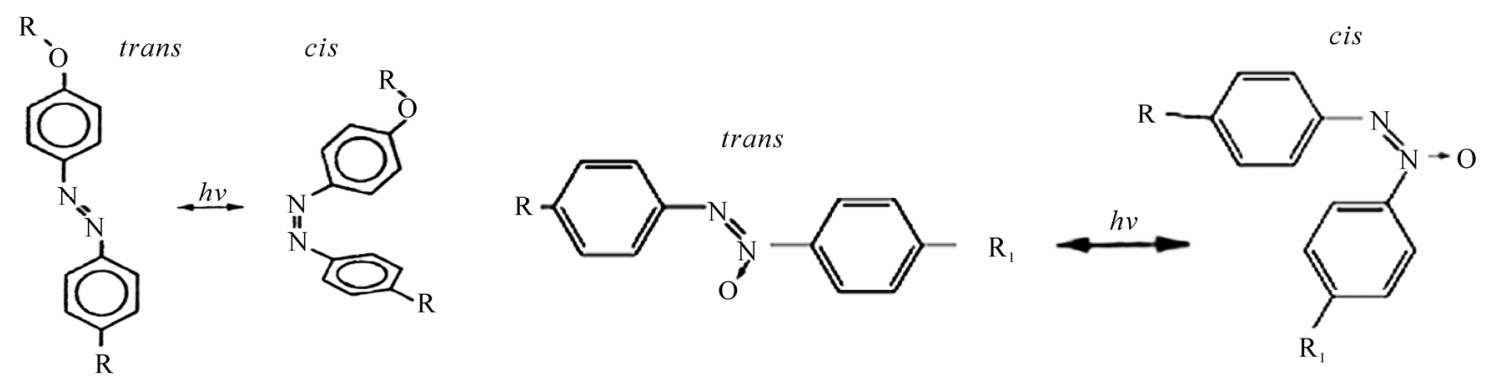

Figure 2. Scheme of of photoisomerization of azo- and azoxy-benzene compounds.

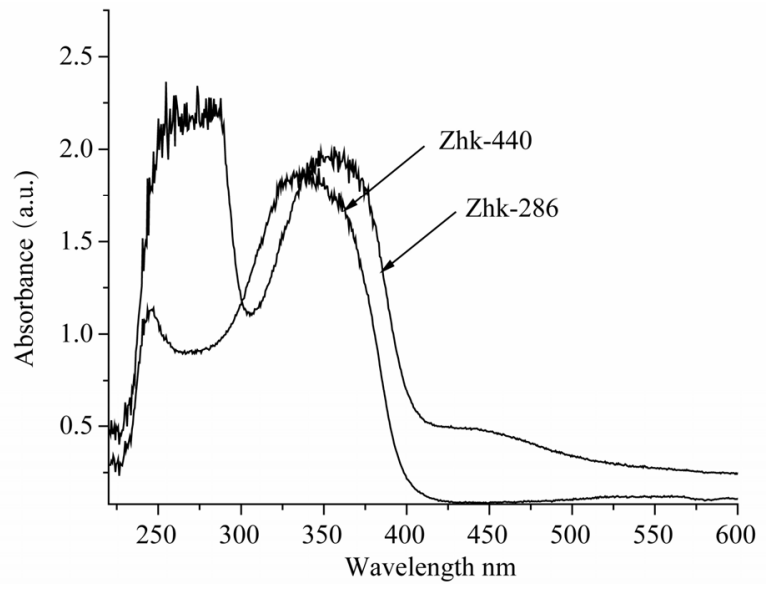

Figure 3. Absorption spectra of the azo- (ZhK 286) and azoxy- (ZhK 440) compounds.

$320 \mathrm{~nm}$ ) and thus is able to precisely measure UV irradiation dose in accordance with the Erythemal Response Spectrum. The properties of this sensor are shown in Figures 4-6.

The idea of tunable lasing in CLCs was expressed in patent of Goldberg and Shnur [25]. The theoretical analysis performed by Kukhtarev [26] demonstrated that the observation of lasing with a distributed feedback in a cholesteric liquid-crystal mixture containing a luminescent dye requires a pump power of $\sim 10^{5} \mathrm{~W} / \mathrm{cm}^{2}$. Il'chishin et al. [27] were the first to design a dye-doped cholesteric liquid-crystal laser. More recently, the consideration of chiral LCs as a photonic gap medium promoted explanation of the appearance of laser emission near the edge of the selective reflection band [28] and also stimulated the investigation of this emission. This approach allowed those authors to explain the observed laser emission at the edge of the selective reflection band (SRB) in the DD CLC. Within the band gap, the wave exponentially decays when propagating deep into the crystal and, correspondingly, the density of states in the band gap becomes considerably lower. Since the degree of spontaneous emission according to the Fermi law is proportional to the number of photon states, the spontaneous emission is suppressed within the band gap and, accordingly, in-

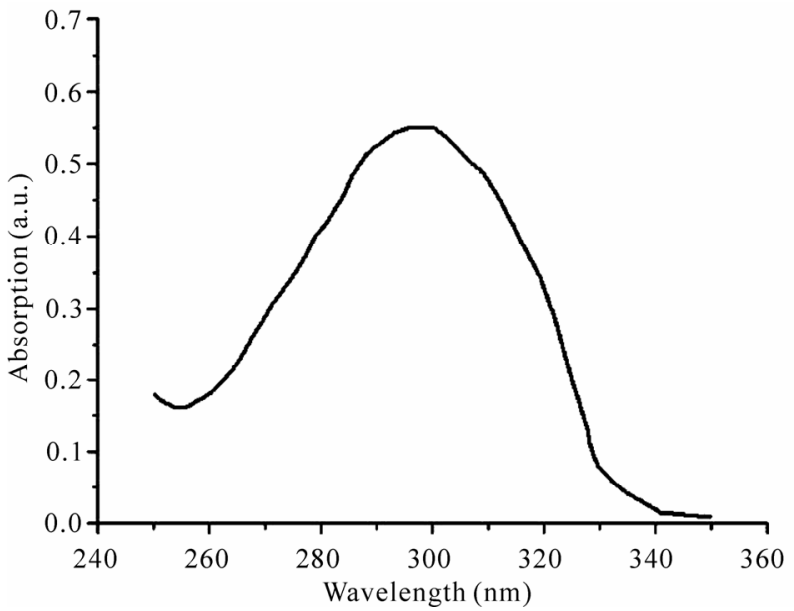

Figure 4. Absorption spectrum for a mixture with $99 \%$ of an UV transparent nematic and $1 \%$ of 4-cyanophenyl ester of 4' heptylcinnamic acid (Zhk-537, NIOPIK).

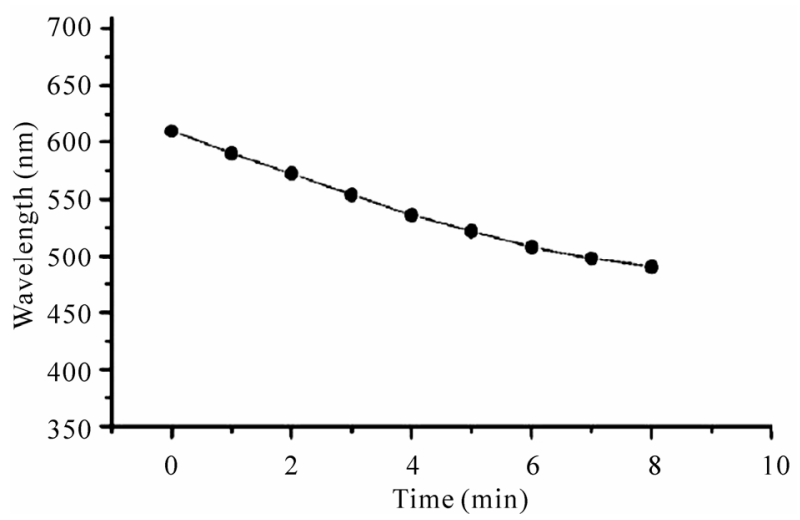

Figure 5. The selective reflection wavelength as a function of UV radiation time for the mixture $76.5 \%$ (50\%ZLI-1695 + 50\%Zhk-537) + 23.5\%ZLI-6248.

creases at the band edges. A comprehensive analysis of the CLC as a photonic crystal is given in the review by Kopp et al. [29].

Tunability of the lasing in these systems can be achieved by the change of chiral dopant concentration, by temperature variation, by mechanical stress or applying an electric field. 


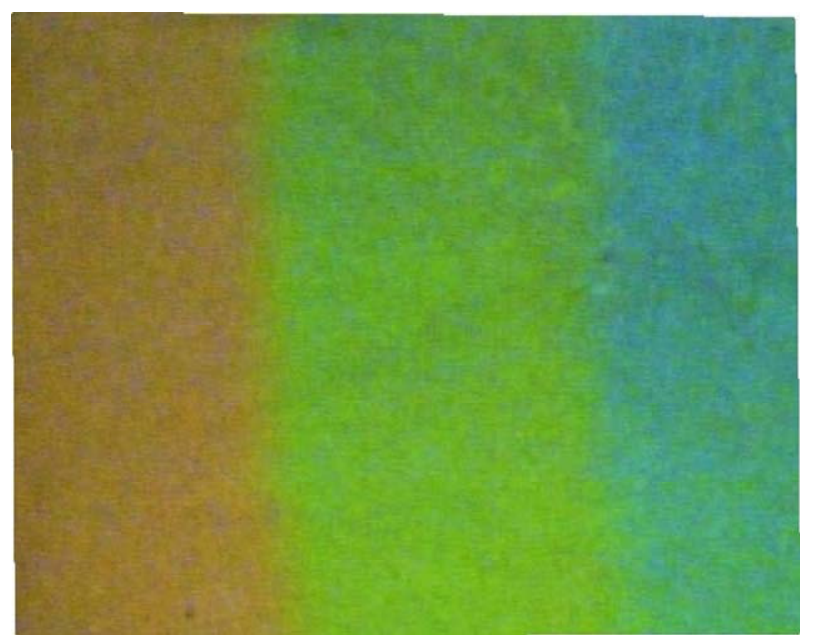

Figure 6. Change of color under direct sun irradiation. (76.5\% (25\%ZLI1695 + 75\%ZhK537) + 23.5\% MLC- 6248). Gold - no UV exposure. Green - 4 min direct sun irradiation. Blue $-8 \mathrm{~min}$ irradiation.

In [30] was proposed the tuning of the lasing wavelength by light. In this pioneering work the controlling of the generated laser wavelength was carried out by means of phototransformation of a well known chiral dopant from Merck ZLI-811 [left-handed (S-811) and righthanded ZLI-3786 (R-811)] whose light sensitivity was never considered before. The experimentally observed phenomenon of photo-transformation of the OAD ZLI811 under UV irradiation is explained by the photo-Fries rearrangement of its chiral molecules, which is usually observed in complex aromatic esters. The Fries rearrangement (Figure 7), which is irreversible, can be induced photo-chemically or chemically $[31,32]$.

The absorption spectrum of ZLI-811 solved in the transparent nematic ZLI-1695 is shown in Figure 8.

The dependence of the selective transmission on the irradiation time of ZLI-811 solved in the transparent nematic mixture ZLI 1184 + ZLI 1185 (cyclohexanes, Merck) is shown in Figure 9. The samples were irradiated by light from a Mercury lamp with a 240 - to 400 $\mathrm{nm}$ filter.

In order to test the phototuning of the laser emission, a 40-mm-thick cell was prepared with a pitch that provides laser emission at $382 \mathrm{~nm}$, in correspondence of the long wavelength edge. The cholesteric mixture was prepared mixing a nematic ZLI-1695 $66 \mathrm{wt} \%$ and left-hand chiral dopant ZLI-811 $34 \mathrm{wt} \%$. $0.5 \mathrm{wt} \%$ of a photoluminescent dye 4-cyano-4"-decyloxy-p-terphenyl

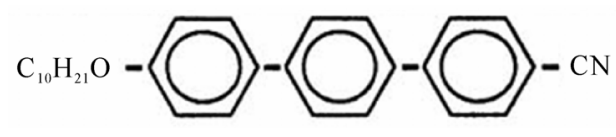

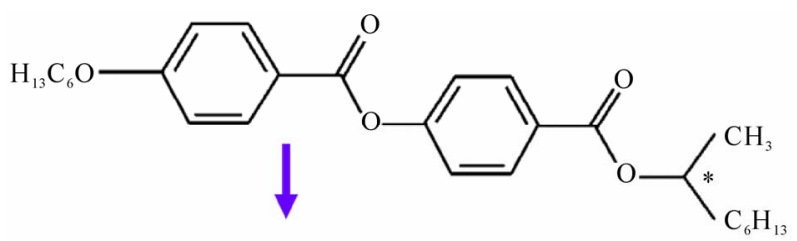

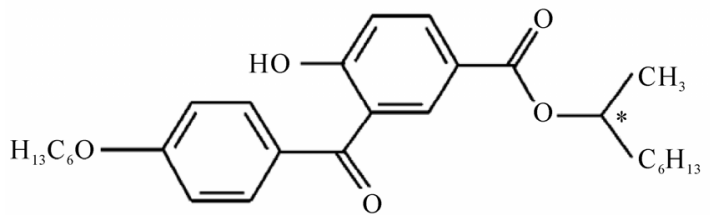

Figure 7. Photo-fries rearrangemen.

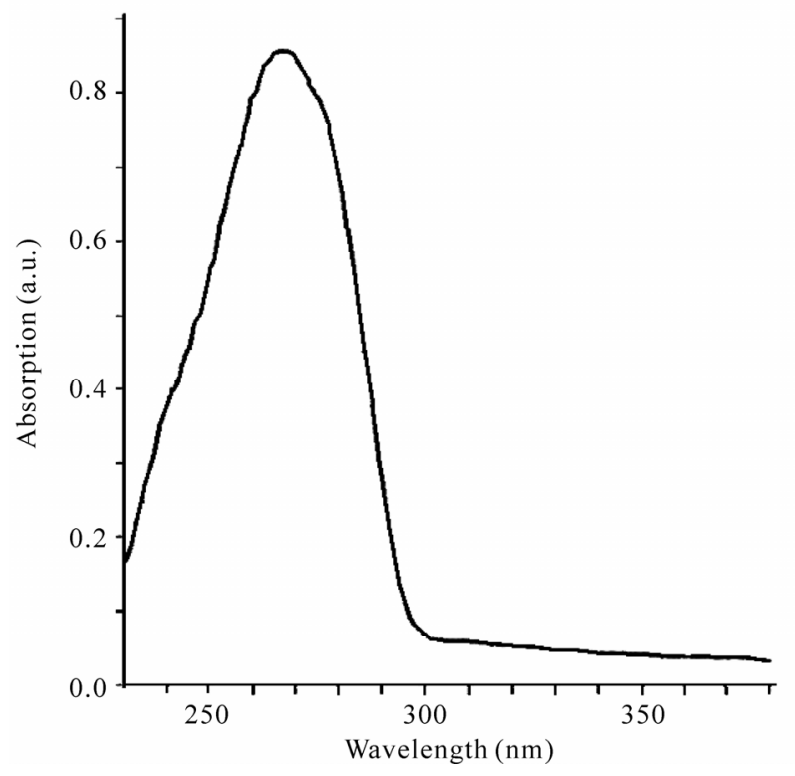

Figure 8. Absorption spectrum of $1 \%$ solution of S-811 in ZLI 1695.

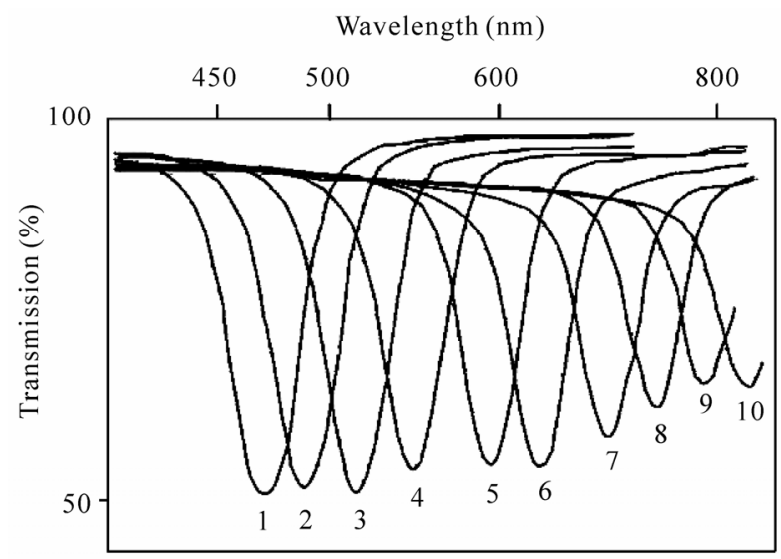

Figure 9. Transmission spectra of the $36 \%$ ZLI-1184 + 36\% ZLI-1185 + 28\% ZLI-811 mixture irradiated for different times. 1: Before irradiation, 2: 3 min or irradiation, 3: 7 min, 4: $11 \mathrm{~min}$, 5: $16 \mathrm{~min}$, 6: $21 \mathrm{~min}$, 7: $26 \mathrm{~min}$, 8: $30 \mathrm{~min}$, 9: $34 \mathrm{~min}, 10: 37 \mathrm{~min}$. 
was then added to the mixture. This is a liquid crystalline dye with the following phase sequence: $\mathrm{Cr}-99^{\circ} \mathrm{C}-\mathrm{S}_{\mathrm{A}}$ $227^{\circ} \mathrm{C}=\mathrm{N}-230^{\circ} \mathrm{C}-$ I. The material exhibits a violet emission with a maximum at about $390-400 \mathrm{~nm}$ wavlength.

The scheme of experiment is shown in Figure 10.

The pumping with third-harmonic radiation of a Qswitched Nd:YAG laser (Continuum, Surelite II, $355 \mathrm{~nm}$, $6 \mathrm{~ns}, 5-10 \mathrm{~Hz}$ ) led to laser emission, which could be tuned under exposure to light from a $100 \mathrm{~W}$ mercury lamp with a filter transparent in the range $240-400 \mathrm{~nm}$. After step-by-step light irradiation from the UV lamp, shifts of the lasing wavelength were observed. In Figure 11 laser lines for different exposure times are shown. The total shift encompasses more than $30 \mathrm{~nm}$ wavelength range, from the near UV to the visible. Illuminating the sample over an hour, the laser emission at the long wavelength edge of the band gap vanishes. At this stage, an unstable peak appears on the short edge of the gap, jumping to the long wavelength edge and back.

Since the publication of this work, there have appeared a number of papers concerned with phototunable CLC [33-37]. In [36,37], the reversible tuning was performed using a trans-cis photoisomerization of the chiral azo

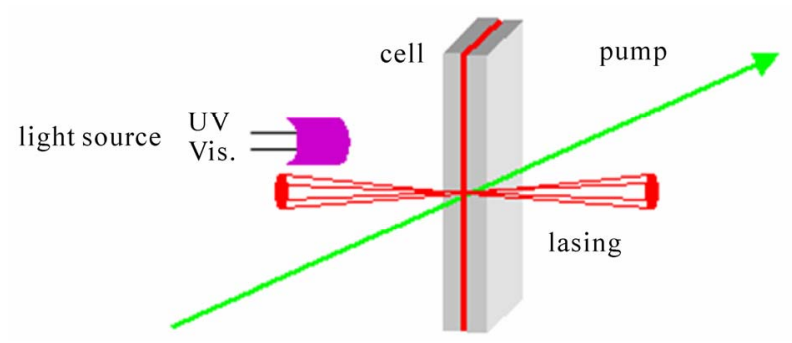

Figure 10. Scheme of the photo tunable CLC laser.

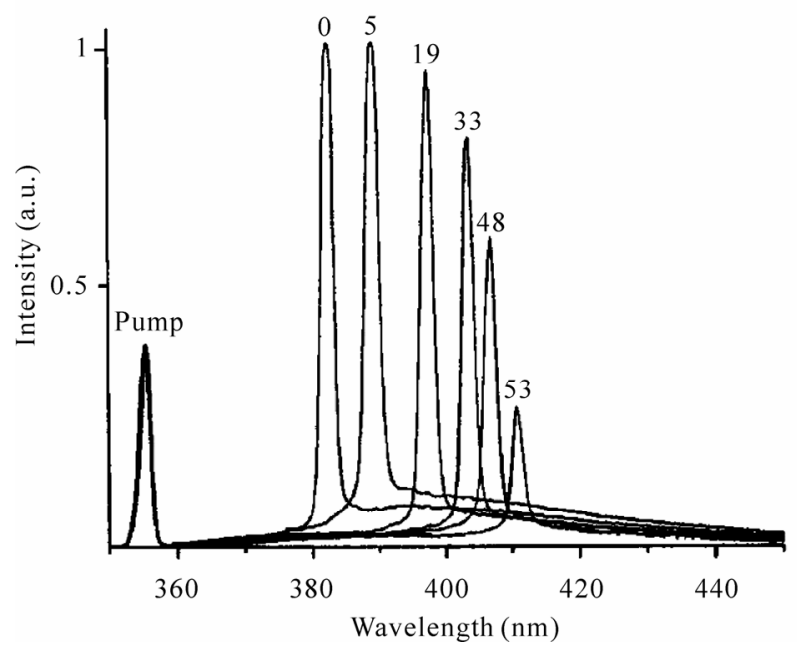

Figure 11. Laser lines from the CLC exposed for several time durations. Numbers indicate the irradiation time in minutes. component of a cholesteric liquid-crystal mixture. Lin et al. [37] achieved a change in the helical pitch up to $110 \mathrm{~nm}$ through exposure to light from an ultraviolet lamp for 20 min. However, the reverse relaxation transition to the initial trans state was observed only within 20 hours.

Note that the high light sensitivity of azo and azoxy compounds made it possible to use Light-Emitting Diodes (LEDs) instead of a mercury lamp in order to control the helical pitch of CLCs. The reversible tuning of the lasing wavelength in CLCs containing nematicazo- or azoxy- photo-sensitive components was accomplished by varying the time of irradiation of samples with the use of two LEDs: at $405 \mathrm{~nm}$ wavelength for blue shift and at $466 \mathrm{~nm}$ wavelength for red shift [38]. The second harmonic signal from a Nd:YAG laser was used as light source for pumping the cholesteric resonators. The pulse wavelength, width, and repetition rate were $532 \mathrm{~nm}, 4 \mathrm{~ns}$, and $1 \mathrm{~Hz}$, respectively.

The investigations indicate that in spite of the fact that the azo compounds were more sensitive to light irradiation, and that the isomerization processes were faster for these compounds than for the azoxy compounds, the azoxy materials were more suitable for use in lasing devices. Furthermore, azoxybenzenebased liquid crystals are transparent over a broader region of the electromagnetic spectrum than azobenzene compounds, which may enable lasing at short wavelengths: for these measurements, a small amount (not more than $2 \%$ ) of the compound was dissolved in a transparent nematic matrix. Finally, the azoxy-compounds were less sensitive than the azo-compounds, and also exhibited better optical properties. The shift in the lasing wavelength for different exposure times is shown in Figure 12 for the mixture $99.5 \%(71 \%$ ZhK- $440+29 \%$ MLC-6247) $+0.5 \% \mathrm{DCM}$; where ZhK-440 is an azoxy-component and the dye is DCM-p-dimetylaminostyryl 4H-pyran, by Exciton.

These experiments demonstrate LED-controlled reversible tuning in both directions, from longer to shorter wavelengths and vice versa, of a DD CLC laser. The use of LEDs to induce photo-transformations and laser tuning represents a very simple but relevant innovation over similar light-controlled tunable CLC laser systems that use UV lamps, primarily because of the possibility of incorporating such small devices inside novel compact laser systems.

\section{Spatially Tunable DD CLC DFB Lasers}

An alternative approach to the generation of laser radiation tunable over a wide range of wavelengths in DD CLCs based on preparation a cell with gradient of the helical pitch was proposed in [39]. The pitch gradient was produced by filling of the cell with a cholesteric liquid-crystal mixture having a concentration gradient of 


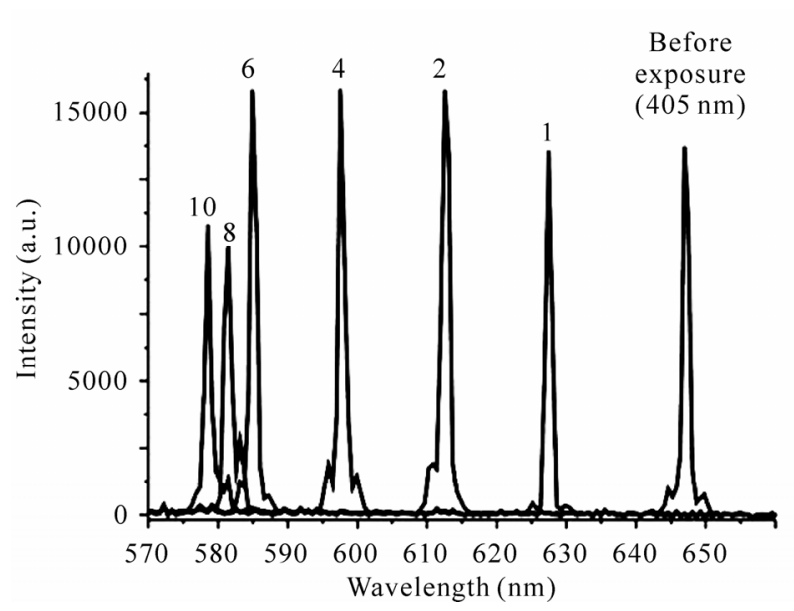

(a)

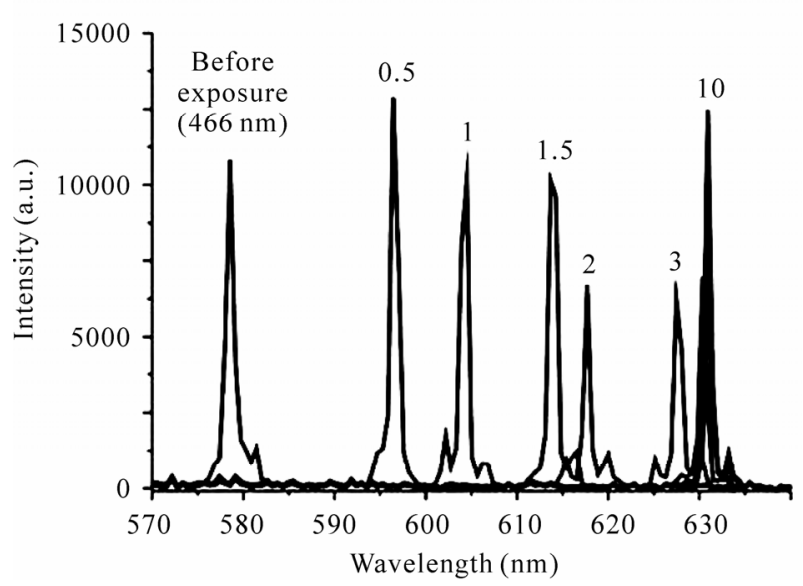

(b)

Figure 12. Lasing spectra of a $99.5 \%(71 \%$ ZhK- $440+29 \%$ MLC-6247) + $0.5 \%$ DCM mixture upon exposure to a 405$\mathrm{nm}$ light emitting diode (a) and after subsequent exposure to radiation of a 466-nm light-emitting diode (b) Numbers indicate the irradiation time in minutes.

chiral dopants. In this way one achieves a continuous tuning of laser emission only moving the CLC cell with respect to the position of the pump laser beam. These experiments have been carried out in CLC mixtures with the nematic ZLI-1695, the chiral dopant ZLI-811, and a terphenyl derivative as dye. Two mixtures with different concentrations of the chiral dopant were prepared: the first one with SRBin red - near-infrared range and the second one with SRB in near UV-violet range. The starting empty cell was half filled by capillarity with the first mixture and the filling was then completed using the second mixture. The pitch gradient was stable for a few months. In order to test the laser emission tuning, the cell was placed on a translational stage that let the sample move orthogonally to the exciting laser beam. The laser action was then investigated at several sample positions.
Figure 13 shows the lasing spectra of the DD CLC obtained for tiny displacement of the cell, at $2 \mu \mathrm{J}$ excitation energy source from the third harmonic of a Nd:YAG laser at pulse wavelength $355 \mathrm{~nm}$. The total shift encompasses more than $30 \mathrm{~nm}$, from about $393 \mathrm{~nm}$ to $427 \mathrm{~nm}$.

The tuning range of laser radiation was limited by the width of the dye emission band. The tuning range can be broadened with the use of the second dye whose absorption band coincides with the luminescence band of the first dye. In this case, there occurs an energy transfer, which is known as the Förster transfer [40]. In [41] the range of laser wavelengths was extended with the use of a specially designed cell by help of developed method of preparing a cell containing several dyes. The CLC mixture contained the following six dyes:

D1 4-cyano-4'-decyloxy-p-terphenyl (ICMA Universidad de Zaragoza, Spain);

D2 2,5-bis (5-tert-butyl-1, 3-benzoxazol-2-yl) tiophene [41];

D3 $N$ - (3-metoxypropyl) -4 (3-metoxypropylamino) -1, 8-naphtalimide [42];

D4 2,3,5,6-1H, $4 \mathrm{H}$-tetrahydro-8-methylquinolizino- (9, 9a, 1gh) (Coumarin 102 from Exciton);

D5 9- (4-decylxoycarbonylphenylethynyl) -10-(4-ethyloxyphenylethynyld antracene (ICMA Universidad de Zaragoza, Spain);

D6 (4-Dicyanomethylene-2-methyl-6-(p-dimethylaminostyryl) -4H-pyran (DCM from Exciton).

The dyes are selected in order to cover a wide wavelength range from UV to visible, and employ the same pump source. D1 has been used for UV luminescence; D2 for violet luminescence, and also in Förster energy transfer combined with D3 for blue-green luminescence. D4 and D5 have been used in Förster transfer for green

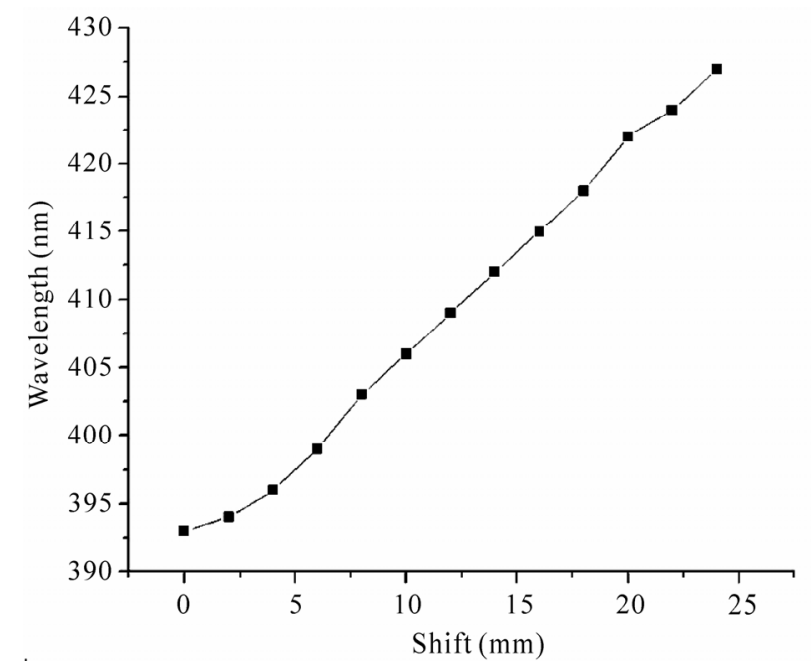

Figure 13. Wavelength tuning of DD CLC laser obtained by shifting the cell with concentration gradient. 
luminescence. Finally, D6 is used for yellow, orange, and red luminescence. The total luminescence band of these dyes covered the range from 373 to $684 \mathrm{~nm}$. As seen from Figure 14 the luminescence spectrum of the blue-2 dye overlaps well the absorption spectrum of the green dye.

As a CLC the mixture of the nematic liquid crystal ZLI-1646 and the optically active dopant MLC-6247 both from Merck was employed. The Nitrogen laser (337 $\mathrm{nm}, 4 \mathrm{~ns}, 1-10 \mathrm{~Hz}$ ) was used as a pump source. Figure 15 shows the lasing spectra of the dye doped CLC at several pitches corresponding to different cell positions, obtained by translation. The total shift covers more than $300 \mathrm{~nm}$ wavelength range, from 370 to $680 \mathrm{~nm}$.

In conclusion, tuning of a DD CLC mirror-less laser has been obtained in one single sandwich cell with several mixtures dye-CLC combined in order to cover almost completely the wavelength range from UV to red, also making a suitable cholesteric pitch gradient inside the cell. The relevant aspect of the method is that a simple translation of the cell with respect to a single exciting beam enables fine tuning of the laser wavelength in a very large spectrum range. Thus, the obtained results demonstrate a possibility of designing compact DD CLC lasers quasicontinuously tunable over a wide range of wavelengths.

Recently, lasing in DFB CLC cells with spatial helical pitch gradient obtained in different ways was shown. A spatially tunable laser emission from DD CLC cell with one-dimensional temperature gradient was proposed in [43]. The lasing wavelength was widely tunable from 577 to $670 \mathrm{~nm}$ by shifting the position of the dye-doped

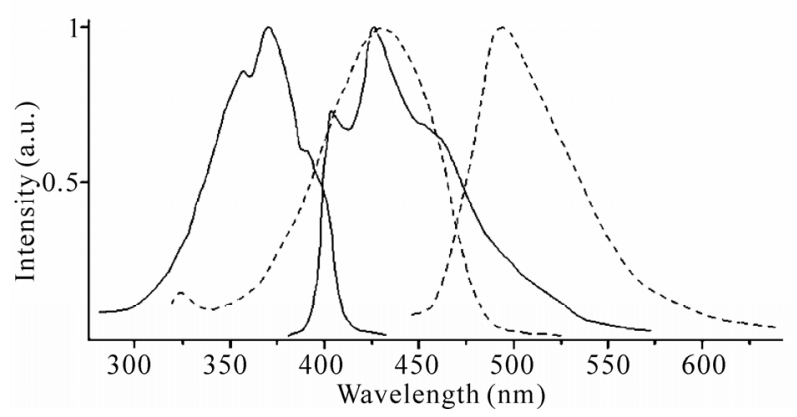

Figure 14. The absorption and emission spectra of blue sensitizer (D2) with solid lines and green emitter (D3) with dashed lines.

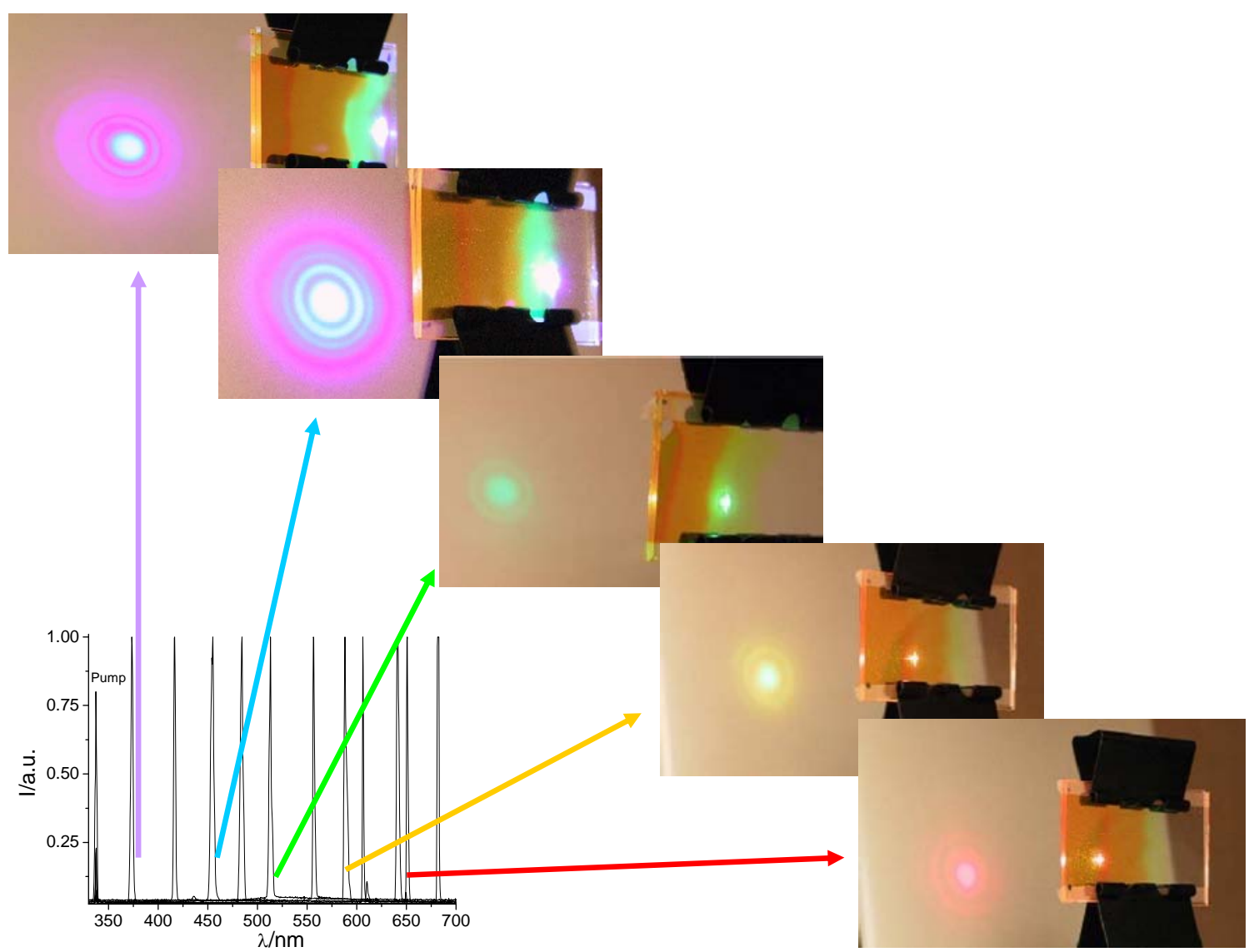

Figure 15. Some laser lines from the DD CLC obtained shifting the cell with respect to the pumping beam. 
CLC cell with respect to the pumping beam. In the other case by introducing short-pitch CLC and Coumarin dye from the left side of an empty cell and long-pitch CLC and two dyes (Coumarin and DCM) from the right side simultaneously a structure with helical pitch and dye concentration gradients was created [44]. Using the Förster energy transfer between two dyes the lasing from the structure continuously covered full visible range from $470 \mathrm{~nm}$ to $670 \mathrm{~nm}$. The tuning was performed by displacement of the cell with respect to the pumping beam. Also a spatial pitch gradient in CLC with two dyes (Förster couple) with a concentration gradient was created by means of a photo-controlled effect [45]. In this case position-sensitive laser emission covering the visible region from $480 \mathrm{~nm}$ to $670 \mathrm{~nm}$ was obtained.

\section{Dye Lasers with CLC Mirrors}

One of the main drawbacks of CLC lasers, limiting their technological application is their low stability. This is connected with two phenomena occurring under the influence of a powerful pumping: gradual deformation of the CLC layer planar orientation and degradation of the luminescent dye molecules. The problem of dye molecules degradation is common to conventional dye lasers as well and, to solve it, the dye solvent is circulating continuously through the laser chamber, avoiding the saturation effects from pumping. Since in DD CLC lasers it is impossible to make the dye circulate separately from the CLC structure, a rotating cell was suggested to avoid deformation of the CLC texture and bleaching of the dye due to the high energy of the pumping beam [46]. This rotating mechanism dramatically increased the stability of the emitted laser beam from several minutes up to several hours.

Several attempts were made to optimize the lasing conditions and performance characteristics of DD CLC. Enhanced laser emission is demonstrated by incorporateing a single external CLC reflector in a DD CLC laser [47].

Another way to enhance the lasing property of DD CLC systems is the introduction of defects in the cholesteric structure. In this case lasing is observed inside the band gap. The introduction of a defect into the CLC can be achieved in two ways: replacing a part of the host medium with a material that has a different dielectric constant e.g. two layers of CLC sandwich a thin layer of an isotropic medium [48]; introducing a phase jump inside the CLC cell [49]. The introduction of a defect and the tuning of photonic defect modes in the CLC, by means of the local deformation of the helix in the middle of the CLC layer, were considered theoretically in [50]. Experimentally the defect mode emission was investigated mostly in polymeric CLCs. Defect mode lasing was ob- served in a DD cholesteric polymeric network [51], where the defect was produced by a phase jump of the cholesteric helix at the interface of two staked layers of polymer film.

The lasing in three layers cell where dye solution is sandwiched between two CLCs can be considered as defect mode type. Diffrerent dye solutions were investigated: in three layered structures where between two CLC layers with the same pitch and handedness a dye doped nematic LC [52] or a dye doped CLC [53] was sandwiched. Also the defect mode type lasing was studied when between dielectric multilayers a dye doped CLC [54] or a dye doped nematic LC [55] was sandwiched. It should be noted that the lasing threshold observed with the three-layered helical CLC is lower than for conventional DD CLC lasers $[54,56]$.

In this paragraph the investigations of lasing in a multilayer system consisting of a dye doped isotropic solvent sandwiched between two CLC cells will be considered [57-60]. The separation of the CLC and the active medium allows: 1) To avoid the degradation of the CLC structure caused by the absorption of the pumping energy, 2) To use dyes not soluble in LCs and 3) To use the optimal thickness both for the CLC layer and for the dye solution layer (thicker dye layer and thinner CLC layer). CLC mixture was prepared by mixing a nematic MLC6816 (Merck, cyclohexylcyclohexanes) with right handed chiral MLC-6248 (Merck). Not soluble in LCs Rhodamine-6G was used as a dye and glycerol was used as an isotropic solvent. In Figure 16 the scheme of three layer structure is shown.

The second harmonic of a Q-switched Nd: YAG laser (Continuum, Surelite II) was used as a pumping light source. The pulse wavelength, width, and repetition rate were $532 \mathrm{~nm}, 4 \mathrm{~ns}$, and $1 \mathrm{~Hz}$, respectively.

At the beginning of investigations we employed three layered structures with CLC layers possessing equal pitches [57]. In this case typical defect mode lasing was expected. Indeed, as shown in Figure 17 multimode lasing inside the stop band with several emission peaks was observed.

To achieve single-mode lasing the CLC cells were filled with two distinct cholesteric mixtures, whose pitches were shifted in such a way that only the edges of the band gaps overlapped. In Figure 18 the transmission spectra of each CLC layer, the spectra of dye solution emission and lasing in this cell are shown. A single mode lasing occurs in the overlapping part of CLC band gaps.

In contrast with the conventional dye lasers with usual dielectric mirrors, in this kind of CLC lasers the wavelength of lasing is strongly connected with the CLC pitches. By choosing other CLC pitches, one obtains lasing at another wavelength. 


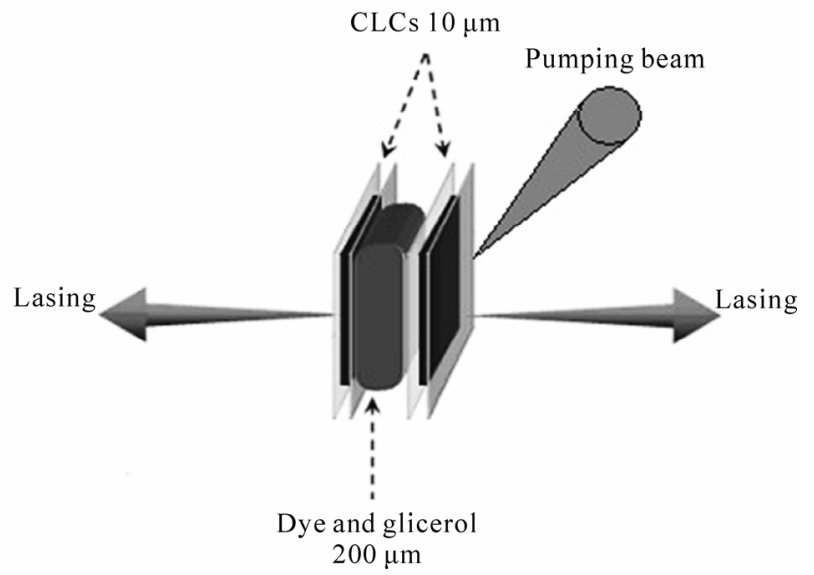

Figure 16. Scheme of the sandwich cell.

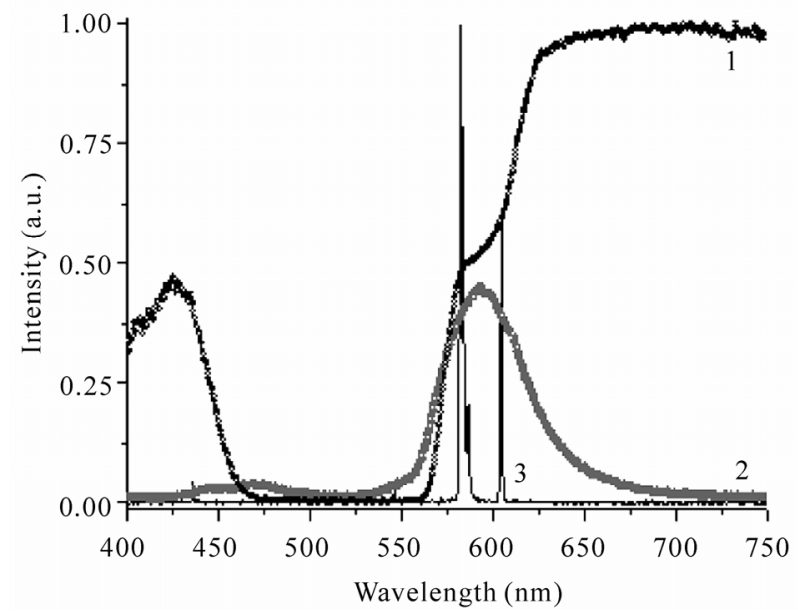

Figure 17. Transmission spectrum of the cholesteric (1), fluorescence of the dye (2) and lasing spectrum from the sandwich cell (3).

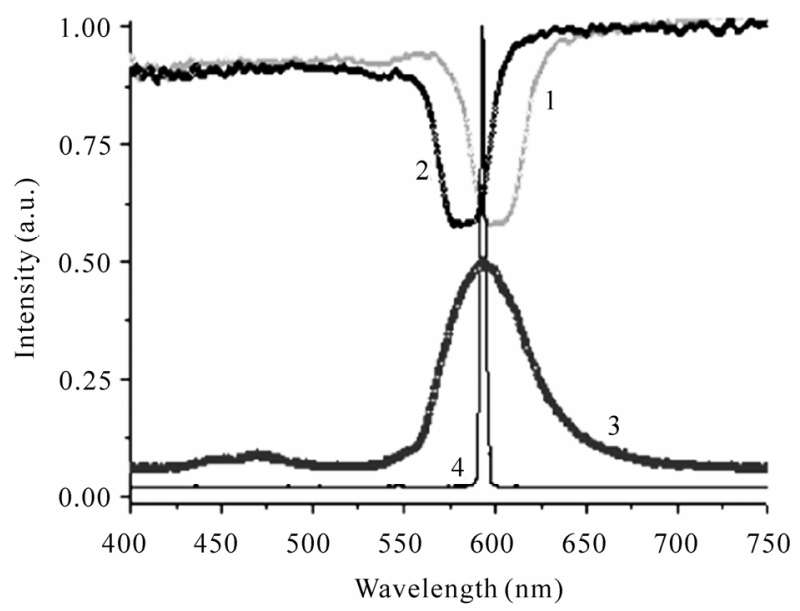

Figure 18. Transmission spectra of two cholesterics with different pitches (1 and 2), fluorescence of the dye (3) and lasing spectrum from the sandwich cell (4).
Besides, Rhodamine-6G, another dye, Stilbene-420, whose absorption and emission peaks are located in UV and violet ranges, was exploited as well. In this case, the CLC pitches were set to get lasing in violet spectrum range and a Nitrogen laser ( $337 \mathrm{~nm}$ ) was used for pumping. Multimode lasing in the structures with equal CLC pitches and single mode one in the shifted pitch configuration were observed, in violet spectrum range and a nitrogen laser $(337 \mathrm{~nm})$ was used for pumping. Multimode lasing in the structures with equal CLC pitches and single mode one in the shifted pitches configuration were observed confirming the general behavior.

The next step of our investigations was obtaining the wide range tunable laser emission in this multilayer system [58-60]. Two approaches for this purpose were exploited: spatially tunable and temperature tunable systems.

In the first case the assembled sandwich cell was composed by a first cholesteric layer containing a material with a well defined pitch and a second cholesteric layer containing chiral materials with different pitches. The CLC layer with invariable pitch contains a wide band gap material while the second layer consists of narrow band gap material with spatially changing pitch. The parallel combination of these two layers forms a spatially tunable optical resonator. A different laser wavelength is emitted from different regions of the cell under the pumping beam irradiation. To obtain the wide photonic band gap layer, BL-006 or alternatively BL-090, with $\Delta \mathrm{n}$ approximately 0.3 , as nematic compounds and MLC6248 as optically active dopant have been used. To prepare the second cholesteric layer, MLC-6816 as a nematic compound, ZLI-3786 as a photosensitive chiral compound and RM-257 as photo- polymer (all the above materials supplied by Merck), the Irgacure 2100 (Ciba) as photoiniziator, as an isotropic solvent glycerol and as luminescent dyes ADS680HO (American Dye Source), not soluble in liquid crystals have been used.

The pitch gradient across the cell with narrow band gap was achieved using two different methods for different CLC mixtures. In first case to the nematic MLC6816 was doped with a chiral compound ZLI-3786 whose chemical structure is identical to the one of ZLI-811. Like ZLI-811 the mixture NLC + ZLI-3786 undergoes a photo-Fries transformation $[30,39]$ if irradiated at wavelengths shorter than $300 \mathrm{~nm}$. To create a pitch gradient within the CLC layer containing ZLI-3786, distinct regions of the layer have been exposed to UV for different times using a customized mask. The addition to the mixture of a photopolymer and a photoiniziator is needed to stabilize the cholesteric structure. The used illumination times have been from 1 up to 5 minutes, to have the helical pitch varying continuously within the second cholesteric layer. Figure 19 shows the transmission spec- 
trum for both wide and narrow band gap cells.

In order to test the laser tuning, the cell has been placed on a translation stage and the sample has been moved with respect to the pumping laser beam in the plane of the CLC layer. Using the dye ADS680HO a laser emission in the near infrared at $790 \mathrm{~nm}$ has been observed for the first time. The laser emission wave-length shift obtained shifting the cell is shown in Figure 20.

The second strategy to obtain the modulation of the photonic band gap position of the second chiral layer relies on the use of a series of CLC mixtures in which the chiral compound concentration changes. Three mixtures with different concentrations of the optically active

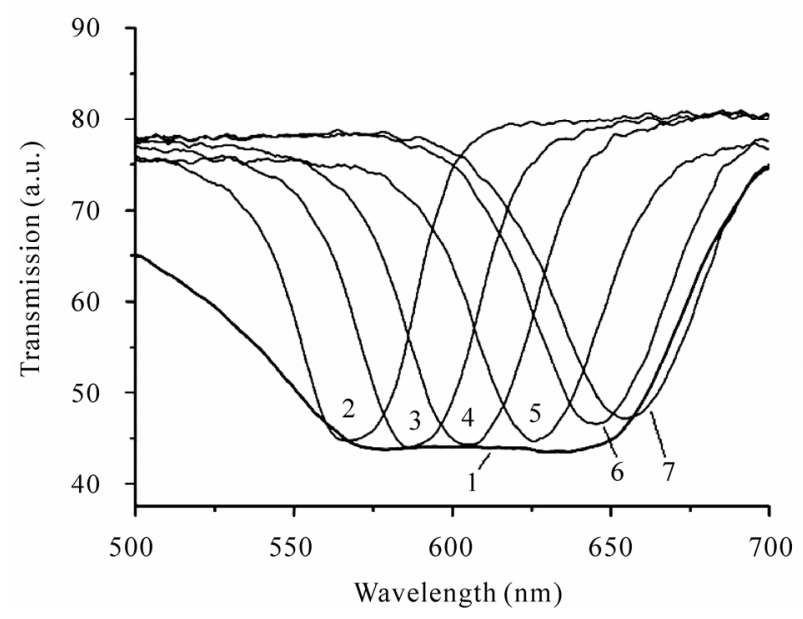

Figure 19. Transmission spectra of the wide band gap cholesteric (1) 75\%BL-006 + 25\%MLC-6248 and of the narrow band gap cholesteric mixture 99\% (74\%MLC-6816 + $26 \%$ ZLI-3786) + 1\% (99\%RM-257 + 1\%Irgacure 2100) after 0 min (2), 1 minute (3), 2 min (4), 3 min (5), $4 \mathrm{~min}$ (6) and 5 min (7) of exposure to a UV lamp.

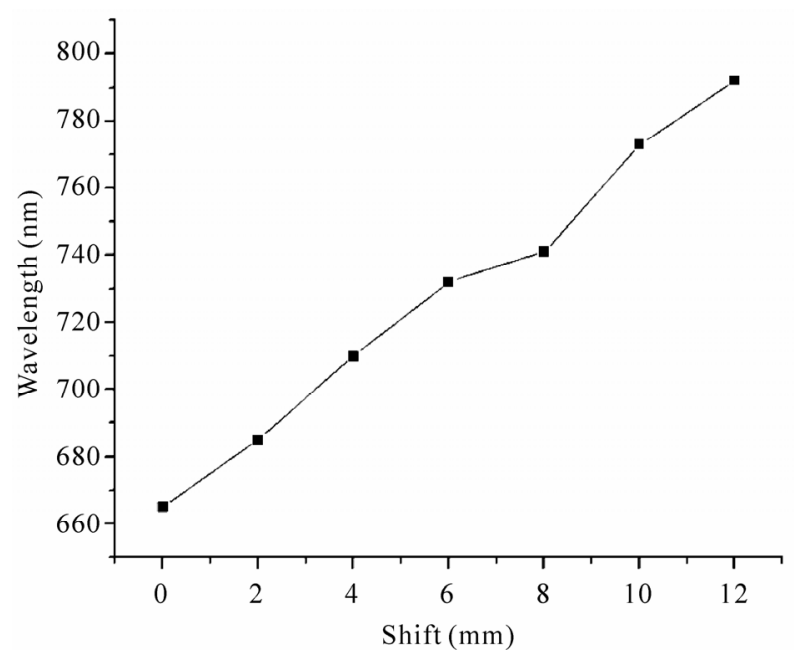

Figure 20. Wavelength tuning of the three-layer laser obtained by shifting the sample. dopant have been prepared. The cell has been partially filled, by capillarity, with one of the three mixtures; filling has been then completed using in sequence the other two mixtures. After assembly, different wavelengths of the visible range are selectively reflected by the whole system. The tuning of the laser emission, from UV to near IR, was observed in different cells using different luminescent dyes. Figure 21 shows the wavelength tuning of laser emission obtained from three different cells. Luminophore OF(mb) (ICMA Universidad de Zaragoza, Spain) used in the first cell is an oligomer with chiral 2-(S)-methylbutyl pendant chains [61].

Also we studied the temperature controllable tuning of lasing in these three layered structures. Owing small birefringence $(\Delta n)$ and high temperature sensitivity and variety of temperature dependence of the pitch the mixtures of cholesteryl ethers are suitable to obtain temperature tunable single mode lasing. Several years ago J. Adams, W. Haas, and J. Wysocki invesigated temperature and concentration characteristics of the pitch of twocomponent cholesteryl chloride and a different carboxylic ester [62]. Varying concentration of components in these mixtures, the spectral range, the sign of $d p / d t$ and slope of $d p / d t$ can be controlled. To obtain tunable lasing, a two component mixture: cholesteryl chloride (24\%) + cholesteryl pelargonate $(76 \%)$ with CLC temperature range $30^{\circ}-75^{\circ}$ and positive temperature dependence of the pitch $(d p / d t>0)$ was selected. In this mixture the selective reflection appears in the region from $560 \mathrm{~nm}$ to 600 $\mathrm{nm}$. A three layer cell, where the central part filled with a solution of glycerol and Rhodamine $6 \mathrm{G}$, was sandwiched between two external layers of CLC, was assembled as shown in Figure 22.

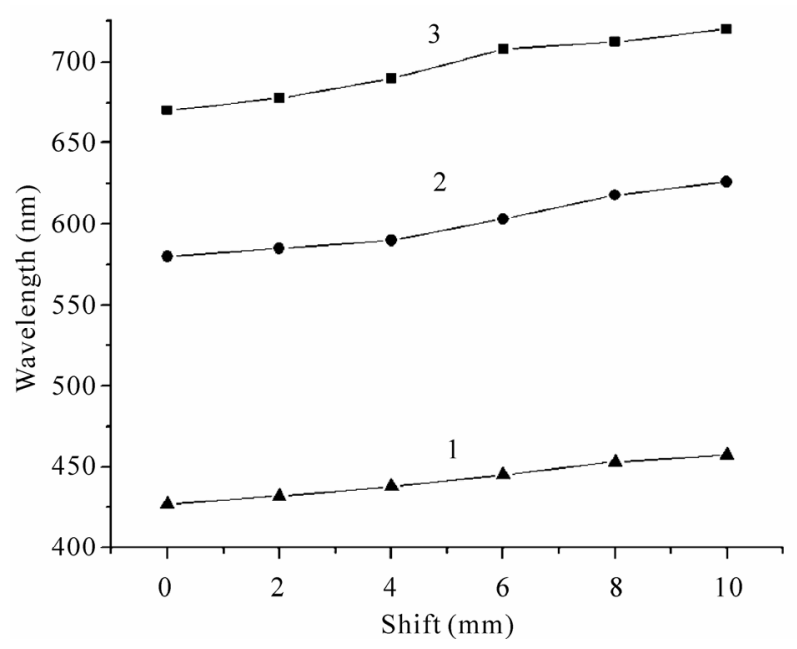

Figure 21. Wavelength tuning of laser emission from three different cells filled with solution of glycerol and the luminophore OF(mb) (1), Rhodamine 6G (2), and LDS698 (3). 
Figure 23 shows the transmission of the cholesteric mixture and luminescence spectrum of $0.4 \%$ Rhodamine $6 \mathrm{G}$ solution in glycerol.

To obtain the laser effect and the tuning of the emitted

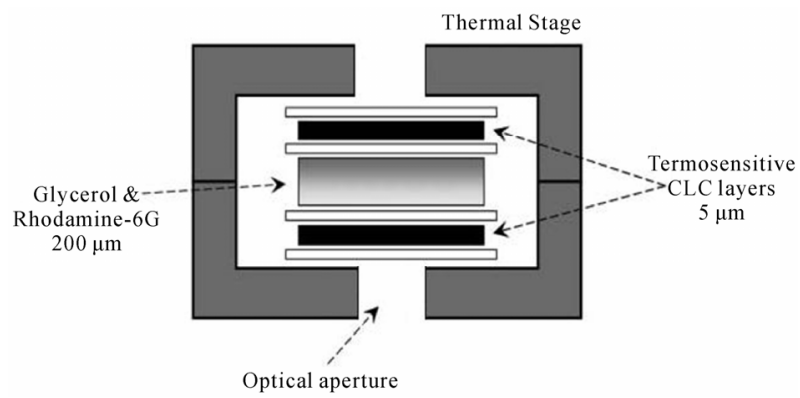

Figure 22. Sketch of the three layer cell in the set-up for temperature tuning of the laser.

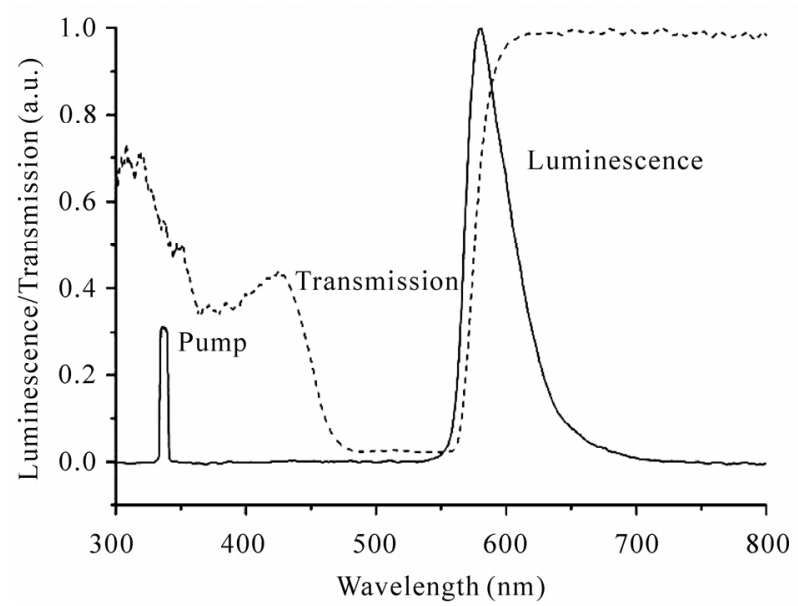

Figure 23. Transmission of the cholesteric mixture [24\% cholesteryl chloride $+76 \%$ cholesteryl pelargonate] (dashed line) and luminescence spectrum of $0.4 \%$ Rhodamine-6G solution in glycerol (solid line).

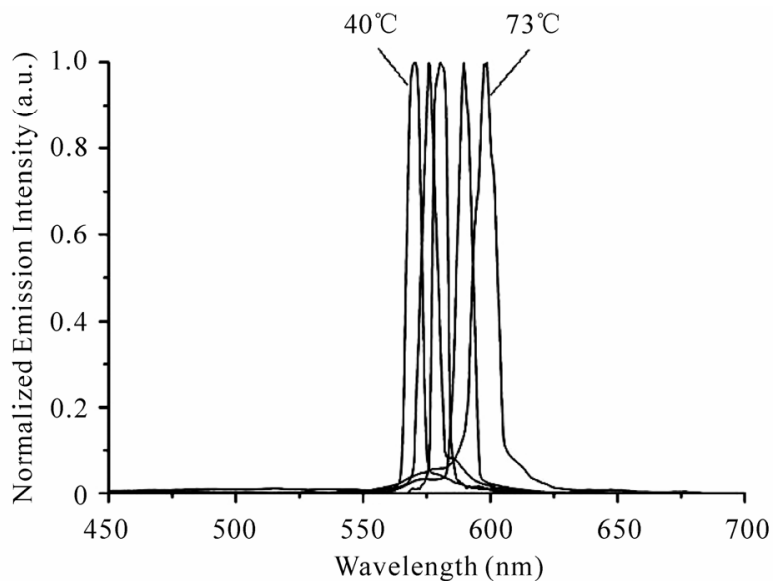

Figure 24. Tuning of the laser peaks obtained by changing the temperature of the three layer structure. wavelength, the three layer cell was fitted in a thermal stage (Figure 22) and placed in front of the pumping beam. The pumping light source was a Nitrogen laser (VSL-337ND-S). The pulse wavelength, width, and repetition rate were $337 \mathrm{~nm}, 4 \mathrm{~ns}$, and 1-10 Hz, respectively. The tuning of the emitted laser wavelength was achieved by varying the temperature of the entire cell. Increasing the temperature from $40^{\circ} \mathrm{C}$ to $73^{\circ} \mathrm{C}$, a shift of the laser peak of about $40 \mathrm{~nm}$ towards longer wavelengths was observed (Figure 24).

To summarize, we have demonstrated recent advances in obtain widely tunable lasing from ultraviolet wavelengths, through the visible range, to infrared wavelengths by employing cholesteric liquid crystals in dye lasers [63].

\section{REFERENCES}

[1] P. G. de Gennes and J. Prost, "The Physics of Liquid Crystals," Clarendon, Oxford, 1993.

[2] S. Chandrasekhar, "Liquid Crystals," Cambridge University Press, Cambridge, 1992.

[3] V. P. Bykov, "Spontaneous Emission in a Periodic Structure," Soviet Journal of Experimental and Theoretical Physics, Vol. 35, 1972, pp. 269-273.

[4] E. Yablonovitch, "Inhibited Spontaneous Emission in Solid-State Physics and Electronics," Physical Review Letters, Vol. 58, No. 20, 1987, pp. 2059-2062. doi:10.1103/PhysRevLett.58.2059

[5] P. P. Sorokin and J. R. Lankard, "Stimulated Emission Observed from an Organic Dye, Chloro-Aluminum PhthaloCyanine," IBM Journal of Research, Vol. 10, No. 2, 1966, pp. 162-163. doi: $10.1147 / \mathrm{rd} .102 .0162$

[6] F. P. Schäfer, W. Schmidt and J. Volze, "Organic Dye Solution Laser," Applied Physics Letters, Vol. 9, 1966, pp. 306-309. doi.10.1063/1.1754762

[7] I. P. Ilchishin, E. A. Tikhonov, V. G. Tishchenko and M. T. Shpak, "Tuning of the Emission Frequency of a Dye Laser with Bragg Mirror in the Form of a Cholesteric Liquid Crystal," Soviet Journal of Quantum Electronics, Vol. 8, 1978, pp. 1487-1488. doi:10.1070/QE1978v008n12ABEH011397

[8] G. Chilaya, "Cholesteric Liquid Crystals: Optics, Electrooptics, and Photooptics," In: H.-S. Kitzerow and C. H. Bahr, Eds., Chirality in Liquid Crystals, Series Partially Ordered Systems, Springer Verlag, New York, 2001, pp. 159-185. doi:10.1007/0-387-21642-1 6

[9] G. S. Chilaya, "Light-Controlled Change in the Helical Pitch and Broadband Tunable Cholesteric Liquid-Crystal Lasers," Crystallography Reports, Vol. 51, 2006, pp. S108S118. doi:10.1134/S1063774506070169

[10] G. Chilaya, "Induction of Chirality in Nematic Phases," 
Revue de Physique Appliquée, Vol. 16, No. 5, 1981, pp. 193-208.

[11] G. S. Chilaya and L. N. Lisetski, "Helical Twist in Cholesteric Mesophases," Soviet Physics Uspekhi, Vol. 24, No. 6, 1981, pp. 496-510. doi:10.1070/PU1981v024n06ABEH004849

[12] G. S. Chilaya and L. N. Lisetski, "Cholesteric Liquid Crystals: Physical Properties and Molecular-Statistical Theories," Molecular Crystals and Liquid Crystals, Vol. 140, 1986, pp. 243-286. doi:10.1080/00268948608080157

[13] G. S. Chilaya, "Effect of Various External Factors and Pretransitional Phenomena on Structural Transformations in Cholesteric Liquid Crystals," Crystallography Reports, Vol. 45, No. 5, 2000, pp. 944-960.

[14] L. Pohl, "Application in Liquid Crystals in Spectroscopy," In: H. Stegemeyer, Guest Ed., Liquid Crystals, Springer, New York, 1994, pp. 173-193.

[15] A. Chanishvili, G. Chilaya, G. Petriashvili and D. Sikharulidze, "Light Induced Effects in Absorbing Cholesterics," 6th European conference on Liquid Crystals, Halle, 2001, \# 2-P3.

[16] A. Chanishvili, G. Chilaya, G. Petriashvili and D. Sikharulidze, "Light Induced Effects in Cholesteric Mixtures with Azoxycompound Nematic Host, Proceedings of the 10th SID Symposium, Advanced Display Technologies," Minsk, 2001, pp. 42-45.

[17] A. Chanishvili, G. Chilaya, G. Petriashvili and D. Sikharulidze, "Light Induced Effects in Cholesteric Mixtures with a Photosensitive Nematic Host," Molecular Crystals and Liquid Crystals, Vol. 409, 2004, pp. 209-218. doi:10.1080/15421400490431291

[18] A. Chanishvili, G. Chilaya, G. Petriashvili and P. J. Collings, "Trans-cis Isomerization and Blue Phases," Physical Review E, Vol. 71, No. 5, 2005, pp. 051705. doi:10.1103/PhysRevE.71.051705

[19] A. Chanishvili, G. Chilaya, G. Petriashvili, R. Barberi, R. Bartolino, M. P. de Santo, M. A. Matranga and P. Collings, "Light Control of Cholesteric Liquid Crystals Using Azoxy-Based Host Materials," Molecular Crystals and Liquid Crystals, Vol. 453, 2006, pp. 123-140. doi:10.1080/15421400600651641

[20] D. Aronzon, E. Levy, P. Collings, A. Chanishvili, G. Chilaya and G. Petriashvili, "Trans-cis Isomerization of an Azoxybenzene Liquid Crystal," Liquid Crystals, Vol. 34, No. 6, June 2007, pp. 707-718.

[21] S. V. Serak, N. V. Tabiryan, G. Chilaya, A. Chanishvili, and G. Petriashvili, "Chiral Azobenzene Nematics Phototunable with a Green Laser Beam," Molecular Crystals and Liquid Crystals, Vol. 488, 2008, pp. 42-55. doi:10.1080/15421400802240144

[22] A. Chanishvili, G. Chilaya, G. Petriashvili, R. Barberi, R. Bartolino and M. Giocondo, "Cholesteric Mixtures Sensitive to Solar Irradiation," Abstracts of the International Workshop on Bulk-Surface Effects in Liquid Crystals for Photonic Applications, Cagliari, 2002.

[23] A. Chanishvili, G. Chilaya, G. Petriashvili, R. Barberi, R.
Bartolino and M. Giocondo, "Cholesteric Mixtures Sensitive to Solar UV B Irradiation," $7^{\text {th }}$ European Conference on Liquid Crystals, Jaca, 2003, p. 150.

[24] A. Chanishvili, G. Chilaya, G. Petriashvili, R. Barberi, R. Bartolino and M. P. de Santo, "Cholesteric Liquid Crystal Mixtures Sensitive to Different Ranges of Solar UV Irradiation," Molecular Crystals and Liquid Crystals, Vol. 434, 2005, pp. 353-366.

[25] L. S. Goldberg and J. M. Schnur, "Tunable InternalFeedback Liquid Crystal-Dye Laser," US Patent, No. 3,771, 065, 1973.

[26] N. V. Kukhtarev, "Cholesteric Liquid Crystal Laser with Distributed Feedback," Soviet Journal of Quantum Electronics, Vol. 8, No. 6, 1978, pp. 774-776. doi:10.1070/QE1978v008n06ABEH010397

[27] I. P. Ilchishin, E. A. Tikhonov, V. G. Tishchenko and M. T. Shpak, "Generation of a Tunable Radiation by Impurity Cholesteric Liquid Crystals," Journal of Experimental and Theoretical Physics Letters, Vol. 32, 1980, pp. 24-27.

[28] V. I. Kopp, B. Fan, H. K. M. Vithana and A. Z. Genack, "Low-Threshold Lasing at the Edge of a Photonic Stop Band in Cholesteric Liquid Crystals," Optics Letters, Vol. 23, No. 21, 1998, pp. 1707-1709. doi:10.1364/OL.23.001707

[29] V. I. Kopp, B. Fan, Z.-Q. Zhang and A. Z. Genack, "Lasing in Chiral Photonic Structures," Progress in Quantum Electronics, Vol. 27, No. 6, 2003, pp. 369-416. doi:10.1016/S0079-6727(03)00003-X

[30] A. Chanishvili, G. Chilaya, G. Petriashvili, R. Barberi, R. Bartolino, G. Cipparrone, A. Mazzulla and L. Oriol, "Phototunable Lasing in Dye-Doped Cholesteric Liquid Crystals," Applied Physics Letters, Vol. 83, No. 26, 2003, pp. 5353-5355. doi:10.1063/1.1636818

[31] V. G. Syromyatnikov, L. O. Vretik, O. V. Yaroshchuk, Y. A. Zakrevskyy, T. M. Kim, J. H. Jo, J. Y. Kim and S. H. Kim, "Naphthalene Containing Polymers as New Photoaligning Materials for LCs," ILCC2000 International Liquid Crystal Conference N18, Sendai, Vol. 368, 2001, pp. 543-549.

[32] N. Kawatsuki, H. Takatsuka, and T. Yamamoto, "Thermally Stable Photoalignment Layer of a Novel PhotoCrosslinkable Polymethacrylate for Liquid Crystal Display," Japanese Journal of Applied Physics, Vol. 40, No. 3A, 2001, pp. L209-L211. doi:10.1143/JJAP.40.L209

[33] I. P. Ilchishin, O. V. Yaroshchuk, S. V. Gryshchenko and E. A. Shayduik, "Influence of the Light Induced Molecular Transformations on the Helix Pitch and Lasing Spectra of Cholesteric Liquid Crystals," XVI International Conference on Spectroscopy of Molecules and Crystals, Vol. 5507, 2004, pp. 229-234.

[34] A. Fuh, T.-H. Lin, J.-H. Liu and F.-C. Wu, "Lasing in Chiral Photonic Liquid Crystals and Associated Frequency Tuning," Optics Express, Vol. 12, 2004, pp. 18571863. doi:10.1364/OPEX.12.001857 
[35] S. Furumi, S. Yokoyama, A. Otomo and S. Mashiko, "Phototunable Photonic Bandgap in a Chiral Liquid Crystal Laser Device," Applied Physics Letters, Vol. 84, 2004, pp. 2491-2493. doi:10.1063/1.1699445

[36] P. V. Shibaev, R. L. Sanford and D. Chiappetta, "Light Controllable Tuning and Switching of Lasing in Chiral Liquid Crystals," Optics Express, Vol. 13, 2005, pp. 2358-2363. doi:10.1364/OPEX.13.002358

[37] T.-H. Lin, Y.-J. Chen, C.-H. Wu, A. Fuh, J.-H. Liu and P.-C. Yang, "Cholesteric Liquid Crystal Laser with Wide Tuning Capability," Applied Physics Letters, Vol. 86, No. 16, 2005, pp. $161120(1-3)$. doi.10.1063/1.1897439

[38] G. Chilaya, A. Chanishvili, G. Petriashvili, R. Barberi, R. Bartolino, G. Cipparrone, A. Mazzulla and P. V. Shibaev, "Reversible Tuning of Lasing in Cholesteric Liquid Crystals Controlled by Light Emitting Diodes," Advanced Materials, Vol. 19, No. 4, 2007, pp. 565-568. doi:10.1002/adma.200600353

[39] A. Chanishvili, G. Chilaya, G. Petriashvili, R. Barberi, R. Bartolino, G. Cipparrone, A. Mazzulla and L. Oriol, "Lasing in Dye Doped Cholesteric Liquid Crystals: Two New Tuning Strategies," Advanced Materials, Vol. 16, No. 9-10, 2004, pp. 791-795. doi:10.1002/adma.200306542

[40] T. Förster, "Zwischenmolekul are Energiewnderung Und Fluoreszenz," Annalen der Physik, Vol. 437, No. 1, 1948, pp. $55-75$. doi:10.1002/andp.19484370105

[41] A. Chanishvili, G. Chilaya, G. Petriashvili, R. Barberi, R. Bartolino, G. Cipparrone, A. Mazzulla, R. Gimenez, L. Oriol and M. Pinol, "Widely Tunable Ultraviolet-Visible Liquid Crystal Laser," Applied Physics Letters, Vol. 86, 2005, pp. 051107 (1-3). doi.10.1063/1.1855405

[42] B. M. Krasovitskii and B. M. Bolotin, "Organic Luminescent Materials," VCH, Weinheim, 1988.

[43] Y. Huang, Y. Zhou and S. T. Wu, "Spatially Tunable Laser Emission in Dye-Doped Photonic Liquid Crystals," Applied Physics Letters, Vol. 88, 2006, pp. 011107 (1-3). doi.10.1063/1.2161167

[44] K. Sonoyama, Y. Takanishi, K. Ishikawa and H. Takezoe, "Position-Sensitive Cholesteric Liquid Crystal Dye Laser Covering a Full Visible Range," Japanese Journal of Applied Physics, Vol. 46, No. 36, 2007, pp. L874-L876. doi:10.1143/JJAP.46.L874

[45] T. Manabe, K. Sonoyama, Y. Takanishi, K. Ishikawa and H. Takezoe, "Toward Practical Application of Cholesteric Liquid Crystals to Tunable Lasers," Journal of Materials Chemistry, Vol. 18, 2008, pp. 3040-3043. doi: $10.1039 / \mathrm{b} 802461 \mathrm{~h}$

[46] G. Chilaya, A. Chanishvili, G. Petriashvili, R. Barberi, M. P. de Santo and M. A. Matranga, "Enhancing Cholesteric Liquid Crystal Laser Stability by Cell Rotation," Optics Express, Vol. 14, 2006, pp. 9939-9943.
doi:10.1364/OE.14.009939

[47] Y. Zhou, Y. Huang, A. Rapaport, M. Bass and S. T. Wu, "Doubling the Optical Efficiency of a Chiral Liquid Crystal Laser Using a Reflector," Applied Physics Letters, Vol. 87, No. 23, 2005, pp. 231107 (1-3).

[48] Y.-C. Yang, C.-S. Kee, J.-E. Kim and H.-Y. Park, "Photonic Defect Modes of Cholesteric Liquid Crystals," Physical Review E, Vol. 60, 1999, pp. 6852-6854. doi:10.1103/PhysRevE.60.6852

[49] V. I. Kopp and A. Z. Genack, "Twist Defect in Chiral Photonic Structures," Physical Review Letters, Vol. 89, 2002, pp. 033901 (1-4).

[50] T. Matsui, M. Ozaki and K. Yoshino, "Tunable Photonic Defect Modes in a Cholesteric Liquid Crystal Induced by Optical Deformation of Helix," Physical Review E, Vol. 69, 2004, pp. 061715 (1-4).

[51] J. Schmidtke, W. Stille and H. Finkelmann, "Defect Mode Emission of a Dye Doped Cholesteric Polymer Network," Physical Review Letters, Vol. 90, 2003, pp. 083902 (1-4)

[52] M. H. Song, B. Park, K.-C. Shin, T. Ohta, Y. Tsunoda, H. Hoshi, Y. Takanishi, K. Ishikawa, J. Watanabe, S. Nishimura, T. Toyooka, Z. Zhu, T. M. Swager and H. Takezoe, "Effect of Phase Retardation on Defect Mode Lasing in Polymeric Cholesteric Liquid Crystals," Advanced Materials, 2004, Vol. 16, No. 9-10, pp. 779-783. doi:10.1002/adma.200306360

[53] M. H. Song, N. Y. Ha, K. Amemiya, B. Park, Y. Takanishi, K. Ishikawa, J. W. Wu, S. Nishimura, T. Toyooka and H. Takezoe, "Defect Mode Lasing with Lowered Threshold in a Three-Layered Hetero Cholesteric Liquid Crystal Structure," Advanced Materials, Vol. 18, No. 2, 2006, pp. 193-197. doi:10.1002/adma.200501438

[54] Y. Matsuhisa, R. Ozaki, M. Ozaki and K. Yoshino, "Single-Mode Lasing in One-Dimensional Periodic Structure Containing Helical Structure and Defect," Japanese Journal of Applied Physics, Vol. 44, No. 20, 2005, pp. L629-L632. doi:10.1143/JJAP.44.L629

[55] R. Ozaki, T. Matsui M. Ozaki and K. Yoshino, "Electrically Color-Tunable Defect Mode Lasing in One-Dimensional Photonic-Band-Gap System Containing Liquid Crystal," Applied Physics Letters, 2003, Vol. 82, No. 21, pp. 3593-3595. doi:10.1063/1.1577829

[56] Y. Takanishi, N. Tomoe, N. Y. Ha, T. Toyooka, S. Nishimura, K. Ishikawa and H. Takezoe, "Defect-Mode Lasing from a Three-Layered Helical Cholesteric Liquid Crystal Structure," Japanese Journal of Applied Physics, Vol. 46, 2007, pp. 3510-3513. doi:10.1143/JJAP.46.3510

[57] G. Chilaya, A. Chanishvili, G. Petriashvili, R. Barberi, G. Cipparrone, A. Mazzulla, M. P. de Santo, H. Sellame and M. A. Matranga, "SingleMode Lasing in Multilayer Sandwiched Systems Consisting of Cholesteric Liquid Crystals and Dye Solution XV International Symposium on 
Advanced Display Technologies," Proceedings of SPIE, Vol. 6637, 2007, pp. 66370M-1- 66370M-6.

[58] G. Chilaya, A. Chanishvili, G. Petriashvili, R. Barberi, G. Cipparrone, A. Mazzulla, M. P. de Santo, H. Sellame and M. A. Matranga, "Lasing in Three Layer Systems Consisting of Cholesteric Liquid Crystals and Dye Solution," Molecular Crystals and Liquid Crystals, 2008, Vol. 495, pp. 97-105. doi:10.1080/15421400802430521

[59] G. Petriashvili, M. A. Matranga, M. P. de Santo, G. Chilaya and R. Barberi, "Wide Band Gap Materials as a New Tuning Strategy for Dye Doped Cholesteric Liquid Crystals Laser," Optics Express, Vol. 17, 2009, pp. 45534558. doi:10.1364/OE.17.004553

[60] M. A. Matranga, M. P. de Santo, G. Petriashvili, A. Chanishvili, G. Chilaya and R. Barberi, "Frequency Tunable Lasing in a Three Layer Cholesteric Liquid Crystal
Cell," Ferroelectrics, Vol. 395, 2010, pp. 1-11. doi: $10.1117 / 12.742910$

[61] G. Petriashvili, G. Chilaya, M. A. Matranga, M. P. de Santo, G. Cozza, R. Barberi, J. del Barrio, L. S. Chinelatto, L. Oriol and M. Pinol, "Chiral Luminescent Compounds as a Perspective for Cholesteric Liquid Crystal Lasers," Optical Materials, Vol. 31, No. 11, 2009, pp. 1693-1696. doi:10.1016/i.optmat.2009.04.010

[62] J. Adams, W. Haas and J. Wysocki, "Dependence of Pitch on Composition in Cholesteric Liquid Crystals," Physical Review Letters, Vol. 22, No. 3, 1969, pp. 92-94. doi:10.1103/PhysRevLett.22.92

[63] R. Barberi and G. Chilaya, "Strategies for Tunable Cholesteric Lasers," In: L. M. Blinov and R. Bartolino, Eds., Liquid Crystal Microlasers, Transworld Research Network, Trivandrum, 2010, pp. 199-222. 